Article

\title{
A Deep Learning Model of Perception in Color-Letter Synesthesia
}

\author{
Joel R. Bock ${ }^{1}$
}

[1] ${ }^{1}$ San Diego, CA, 92120, USA; sauerkraut@gmail.com

\begin{abstract}
Synesthesia is a psychological phenomenon where sensory signals become mixed. Input to one sensory modality produces an experience in a second, unstimulated modality. In "grapheme-color synesthesia", viewed letters and numbers evoke mental imagery of colors. The study of this condition has implications for increasing our understanding of brain architecture and function, language, memory and semantics, and the nature of consciousness. In this work, we propose a novel application deep learning to model perception in grapheme-color synesthesia. Achromatic letter images, taken from database of handwritten characters, are used to induce synesthesia. Results show the model learns to accurately create a colored version of the inducing stimulus, according to a statistical distribution from experiments on a sample population of grapheme-color synesthetes. The spontaneous, creative mental imagery characteristic of the synesthetic perceptual experience is reproduced by the model. A model of synesthesia that generates testable predictions on brain activity and behavior is needed to complement large scale data collection efforts in neuroscience, especially when articulating simple descriptions of cause (stimulus) and effect (behavior). The research and modeling approach reported here begins to address this need.
\end{abstract}

Keywords: synesthesia; deep learning network; color perception; generative adversarial network; cognitive modeling; character recognition; GPU computing

\section{Introduction}

\subsection{Background}

Synesthesia is a psychological phenomenon where sensory signals become mixed; input to one sensory modality produces an experience in a second, unstimulated modality [1]. For example, the experience of colors may be induced by seeing or hearing digits, letters or words. In "grapheme-color synesthesia", viewed letters and numbers evoke mental imagery of colors. These color associations are involuntary, idiosynchratic and highly consistent over time [2]. The study of synesthesia has implications for furthering our understanding of brain architecture and function, as well as creative processes, language acquisition, and learning and memory performance [3]. The study of consciousness itself may benefit from investigation of synesthesia [4].

Due to the significance of synesthesia across disciplines, the scientific literature is rich with studies aiming to account for the physiological origins and behavioral manifestations. Investigations into cognitive and behavioral aspects and neurological substrates are reviewed in [1] and [5].

Cognitive models have been proposed to identify partitions of cognition and perception in synesthesia. In [6], multiple interconnected pathways of form and color analysis under visual or auditory stimuli are modeled. Symbol and color representation domains interact with semantic identification at higher stages of processing. An important contribution of the model in [6] is explicit description of the levels of inducer processing, from preconscious feature analysis to ultimate production of synesthetic assignment of color to the input.

Descriptions of the neurological basis of synesthesia center on two main theories: the synesthetic brain is (1) cross-activation between color processing area V4 and proximal visual word form area in the fusiform gyrus ([7]); or (2) disinhibited feedback between circuits of bottom-up sensory input and 
higher-level visual areas [8]. Functional neuroimaging studies dynamically localize activated cortical regions in synesthetic perception versus controls [9], and provide valuable insight to validate and continue to develop such theories [10].

Such neuroscientific investigations are now collecting huge amounts of data, even down to the granularity of single neuronal firings. How do cognition and behavior arise from the interactions between activated neurons and assemblies under external sensory input? An ongoing challenge is to uncover causal relationships between "big neural" and "big behavioral" data [11].

\subsection{Deep learning models}

Deep learning facilitates machine learning from large scale data. Complicated, abstract representations of structure in computer vision, language processing, and many other domains can be explored [12], [13].

Hinton demonstrated that multilayer generative models could learn the joint distribution of handwritten digit images and their labels [14]. These deep belief networks learned latent representations of the input in densely-connected hidden layers. Generative models were shown capable of (1) learning low-level features in an unsupervised manner, and (2) learning very large number of parameters without overfitting [14].

Recently, Goodfellow [15] introduced generative adversarial networks (GANs), a general framework for training deep learning networks. GANs eliminate the need for difficult probabilistic computations when learning hidden layer parameters.

The central idea of GANs is to establish competition between two deep network models- the discriminator $(D)$ and the generator $(G)$. $G$ is tasked with generating samples $G(\mathbf{z})$ (drawn from $p_{\mathbf{z}}(\mathbf{z})$ ) that appear to $D$ as having been drawn from the actual distribution $p_{\text {data }}(\mathbf{x})$. $D$ must learn to discern between real and artificial data. In this numerical game, model parameters are optimized alternatively to solve the minimax objective function

$$
\min _{G} \max _{D} V(D, G)=\mathbb{E}_{\mathbf{x} \sim p_{\text {data }}(\mathbf{x})}[\log (D(\mathbf{x}))]+\mathbb{E}_{\mathbf{z} \sim p_{\mathbf{z}}(\mathbf{z})}[\log (1-D(G(\mathbf{z}))]
$$

This formulation has an unique global optimum representing the real data distribution $p_{\text {data }}(\mathbf{x})$ [15]. This is true even when the prior distribution $p_{\mathbf{z}}(\mathbf{z})$ is random noise. Extensions of the GAN framework condition $D$ and $G$ on additional information such as class label [16], [17].

Both $D$ and $G$ are implemented as multilayer convolutional neural networks, as described in Section 2.3.

\subsection{Contribution of present study}

In this work, the generator network $G$ is a model for grapheme-color synesthesia. Achromatic letter images, taken from database of handwritten characters [18], are used to induce synesthesia.

$G$ learns to create a colored version of the inducing stimulus, according to a statistical distribution from experiments on grapheme-color synesthetes [19]. The identity of each symbol determines its concurrent color [8].

The spontaneous, creative mental imagery characteristic of the synesthetic perceptual experience is reproduced by the model.

Thr present study contributes an approach to extract essential mechanisms relating sensory input and processing to behavioral effects in neuro- and cognitive science.

\section{Methods}

\subsection{Handwritten letters database}

Handwritten letter images were extracted from the EMNIST dataset [18]. The raw images are stored as $28 \times 28$ pixels, in 8-bit integer format. A modeling sample comprising the EMNIST uppercase 
letters was constructed using the By_Class subset and annotations. We excluded lowercase letters and numeric digits resulting in 220,304 examples. Counts of individual letters in this sample varied from 2,850 ('K') to $>29,000$ ('O'); qualitatively, moderate variance in handwritten morphological structure for given letters was observed. No balancing of the 26 letter-classes was carried out; the aim here was not to develop a discriminative model for classification. ${ }^{1}$

\subsection{Synesthesia color-letter pairs}

Each grayscale letter image was converted to a 3-channel $(R, G, B)$ image using experimental statistics of perceived colors in grapheme-color synesthesia as reported by Witthoft et al. [19]. The most frequently reported letter-color pairings from a large sample of synesthetes $(n=6,588)$ were used to represent the sample population (c.f. Fig.1 in [19]), recognizing that signficant idiosynchratic differences in color experienced for a given letter exists between individuals. These aggregrate "modal" colors for each letter were used to develop the basic examples for generative colorization model learning in the current study. These pairings are listed in Table 1.

Table 1. Grapheme-color associations of 6,588 synesthetes. The most common color assignment reported for each letter is shown. After [19].

\begin{tabular}{cc}
\hline Color & Letters \\
\hline Red & A, M, R \\
Blue & B, D, T, W \\
Green & E, F, G \\
Yellow & C, L, Y \\
Orange & H, J, K, N, U \\
Purple & P, Q, V \\
White & I, O \\
Black & X, Z \\
\hline
\end{tabular}

\subsection{Numerical implementation}

The conditional GAN model of grapheme-color synesthesia perception was adapted from a deep convolutional neural network (CNN) implementation described in [20]. The generator network encodes the input image by six successive hidden layers, each ouputting a reduced dimensional image relative to the input. Six decoding layers follow; these layers invert the compression operations by deconvolution. The final output of the generator is an image with synthetic colorization. Each compression layer reduces noise while increasing the level of abstraction in representation of the input [21]. The discriminator has similar multilayer convolutions, ulimately outputting a reduced image and probabilities of the input image being real or synthetic. Additional details of the GAN architecture appear in [20]. ${ }^{2}$

We developed code to download, extract and preprocess EMNIST handwritten letters [18]; colorize examples based on synesthesia statistics [19]; train, test, analyze and visualize results of the generative learning process. Deep learning experiments were carried out using the TensorFlow software library controlled by the Python language API [22] ${ }^{3}$. Models were developed on a CPU/GPU-based system (i5-3470 16 GB RAM; NVIDIA GeForce GTX 1050 Ti 4 GB on-board).

Transformations and processing for each training image followed the protocol of [20]: (1) convert example $(R, G, B)$ image (described in Sections 2.1 and 2.2) to CIE Lab color space; (2) present the $L$ (grayscale) channel to the input of the generative model $G$; (3) output synthetic $a, b$ channel data

1 The EMNIST data can be obtained at: https:/ / www.nist.gov/itl/iad/image-group/emnist-dataset.

2 See [20] Appendix 1. Original image colorization GAN adapted here is based on code from the repository: https://github. com/sawhney-kartik/gan-colorization.

3 All computer code developed in this study is available to researchers at: boris@github.com/TBD. 
from $G$, and reconstruct a full 3-channel $(L, a, b)$ color image; (4) present real and artificial images to discriminator $D$; (5) evaluate objective function (Equation 1); (6) backpropagate errors through both networks, updating weights using stochastic gradient descent.

Generative model training was performed for three epochs on a random sample of 125,000 images (total 375,000 iterations) from the EMNIST dataset. Periodically, generated images were converted back to $(R, G, B)$ color space and stored to disk for human observation and post-analysis.

\section{Results}

Results of synesthetic letter colorization by the generative network are presented in Figure 1. The figure panels display (a) typical inputs and (b) corresponding outputs in the very early phases of model training $(<3,000$ iterations). Each panel contains samples for one handwritten letter per column. Iteration count progresses from top to bottom. The top-most row on the right-hand side shows the true modal color-letter pairings from the experimental distribution reported in [19] (Table 1). Letters are presented in random order and are unbalanced; the example count seen for each letter is therefore not uniform.

At this stage of learning, the generative network is not producing realistic colored images matching the true distribution. Several letters begin to appear to align with their synesthetic concurrents, but most others miss the mark. The letters often perceived as either black or white in color-grapheme synesthesia $(\mathrm{I}, \mathrm{O}, \mathrm{X}, \mathrm{Z})$ are already reproduced fairly well.

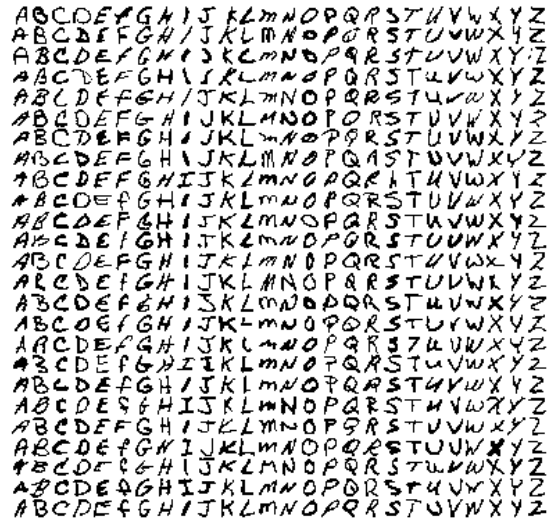

(a) Early generator input.

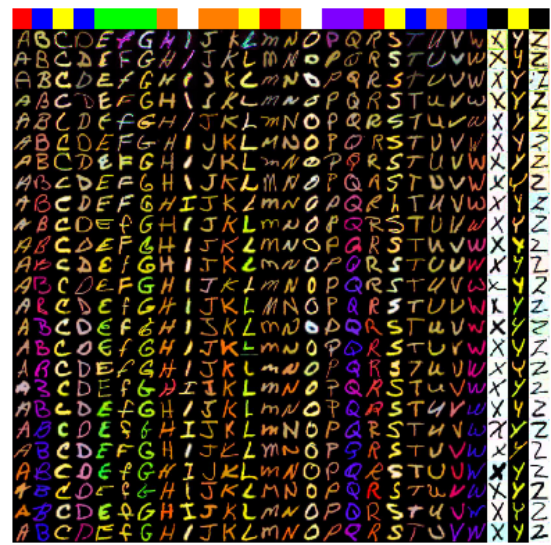

(b) Early generator color induction.

Figure 1. Synesthetic letter colorization by the evolving generative network. Early training: 1st epoch; <3,000 iterations. (a) generator input; (b) generator output. One letter per column. Iteration count progresses from top to bottom.

Following additional optimization of the GAN, more realistic results are observed. Consider the images shown in Figure 2. After three epochs of training, the synesthesia model generates colored letters with high accuracy. The colors produced are nearly indistinguishable from the actual distribution. Accuracy of color reproduction in the 26X26 grid is over $99 \%$; very few instances (G: row 7; F: row 18; K: row 2; O: row 15; Q: row 13; Y: row 9) are incorrectly colored. 
The results exhibited in Figure 2 are typical of those observed in out-of-sample tests on $\sim 95,000$ additional handwritten letter examples. Once the generator learns the true distribution of color-letter associations, the concurrent response remains consistent under additional stimuli.

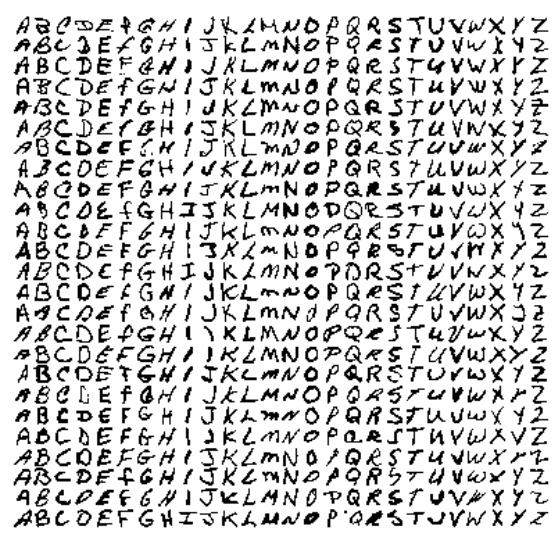

(a) Trained generator input.

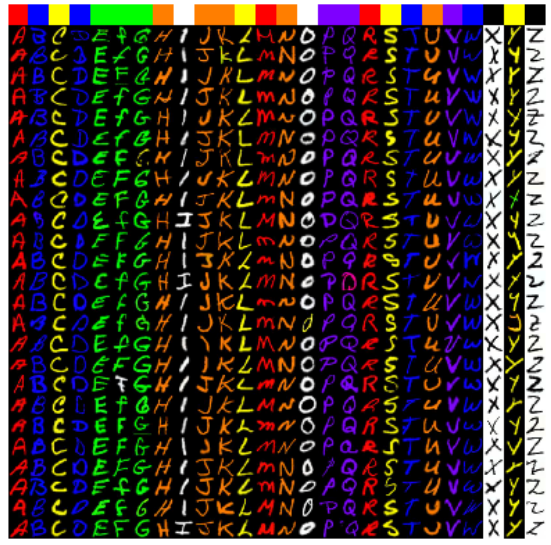

(b) Trained generator color induction.

Figure 2. Synesthetic letter colorization by the trained generative network. Late training: 3rd epoch, $\sim 375,000$ iterations. (a) generator input; (b) generator output. One letter per column. Iteration count progresses from top to bottom.

\section{Discussion}

We applied a generative deep neural network to model perception in grapheme-color synesthesia. Grayscale letters are used to stimulate the model, which colorizes each letter. Training data were taken from experiments on a large sample of synesthetes [19], combined with a database of handwritten letters [18].

Automatic and consistent response to characters or digits are fundamental to synesthesia, even when the stimulus is non-physical (i.e., conceived in the mind's eye) [23]. Recognition of the identity of a symbol determines its concurrent color [8], [2]. In the present model, the generative network hidden layer weights encode information on pixel intensities describing the structure of each letter (and its identity) when perceived in two dimensions. We suggest that a similar mechanism may regulate the process of letter identification and association with color in a synesthete's perceptual experience.

A model of synesthesia that generates testable predictions on brain activity and behavior is needed [2]. With the collection of "big data" in neuroscience, comes an increased complexity of analysis and the distillation of conclusions, especially when articulating simple descriptions of cause (stimulus) and effect (behavior) [11]. The research and modeling approach reported here begins to address this need.

Three particulary ambitious areas in which to apply and extend this research are summarized below.

1. Functional brain imaging. Studies aimed to identify and localize structure and function within the synesthesia experience [5]. Experimental data from functional magnetic resonance imaging (fMRI) or other modalities could be used to build a complementary deep learning model with more explicit mapping of layers to physiologic modular components than used in the current study. Such a model could provide additional insights to refine the competing hypotheses of cross-activation (increased linkage between proximal regions) [7] or disinhibited feedback from higher-level cortical areas [8] in synesthesia. 
2. Language learning and memory. Studying synesthesia may advance our understanding of human perception and information arrangement [6]. One theory proposed in [3] advances the idea that graphemecolor synesthesia develops in part by children to learn category structures; a fundamental task in literacy development is to recognize and discriminate between letters. Therefore synesthesia might arise as an aid to memory. More generally, the ability to discern statistical regularities of printed letters or learn complex rules for letter combinations would assist learning at subsequent stages of literacy development [3]. In [24], small sample experiments suggested that additional sensory dimensions in synesthesia aid in memory tasks when compared to controls. We submit that the generative modeling approach of the current work may be useful to develop and test hypotheses in studies of language acquisition, memory and semantics.

3. Consciousness studies. Synesthesia can give insight into the neural correlates of consciousness, through interaction between sensory inputs and their mediation by semantics in the induction of phenomenal subjective experience [4]. Connecting neural activations with subjective aspects of consciousness (perception of shape, color movement of an object) is potentially achievable following a systematic experimental approach [25]. In deep learning, understanding representations within deep layers is easy at the layer level; at the level of individual neurons, such understanding is much more difficult [26]. Extensions of the deep learning model reported here may help to advance toward these formidable objectives.

Acknowledgments: The author thanks Tom Zingale, who provided the GPU-based computer used to conduct this research.

Conflicts of Interest: The author declares no conflict of interest.

\section{Abbreviations}

The following abbreviations are used in this manuscript:

GAN Generative adversarial network

GPU Graphics processing unit

CPU Central processing unit

CNN Convolutional neural network

fMRI Functional magnetic resonance imaging

\section{References}

1. Hubbard, E.M.; Ramachandran, V. Neurocognitive Mechanisms of Synesthesia. Neuron 2005, 48, 509-520.

2. Mattingley, J. Attention, Automaticity, and Awareness in Synesthesia. Annals of the New York Academy of Sciences 2009, 1156, 141-67.

3. Watson, M.; Akins, K.; Spiker, C.; Crawford, L.; Enns, J. Synesthesia and learning: a critical review and novel theory. Frontiers in Human Neuroscience 2014, 8, 98.

4. van Leeuwen, T.M.; Singer, W.; Nikolić, D. The Merit of Synesthesia for Consciousness Research. Frontiers in Psychology 2015, 6, 1850.

5. Rouw, R.; Scholte, H.; Colizoli, O. Brain Areas Involved in Synaesthesia: A Review. Journal of Neuropsychology 2011, 5, 214-42.

6. Rich, A.; Mattingley, J. Anomalous perception in synaesthesia: A cognitive neuroscience perspective. Nature Reviews Neuroscience 2002, 3, 43-52.

7. Ramachandran, V.; Hubbard, E. Psychophysical investigations into the neural basis of synaesthesia. Proceedings of the Royal Society of London B: Biological Sciences 2001, 268, 979-983.

8. Grossenbacher, P.; Lovelace, C. Mechanisms of synesthesia: cognitive and physiological constraints. TRENDS in Cognitive Sciences 2001, 5, 36-41.

9. Jäncke, L.; Beeli, G.; Eulig, C.; Hänggi, J. The neuroanatomy of grapheme-color synesthesia. European Journal of Neuroscience 2009, 29, 1287-93. 
10. Brang, D.; Hubbard, E.; Coulson, S.; Huang, M.; Ramachandran, V. Magnetoencephalography reveals early activation of V4 in grapheme-color synesthesia. NeuroImage 2010, 53, 268-274.

11. Sejnowski, T.; Churchland, P.; Anthony Movshon, J. Putting big data to good use in neuroscience. Nature Neuroscience 2014, 17, 1440-1.

12. Bengio, Y. Learning deep architectures for AI. Foundations and Trends in Machine Learning 2009, 2, 1-127.

13. LeCun, Y.; Bengio, Y.; Hinton, G. Deep Learning. Nature 2015, 521, 436-44.

14. Hinton, G.E.; Osindero, S.; Teh, Y.W. A Fast Learning Algorithm for Deep Belief Nets. Neural Computation 2006, 18, 1527-1554.

15. Goodfellow, I.; Pouget-Abadie, J.; Mirza, M.; Xu, B.; Warde-Farley, D.; Ozair, S.; Courville, A.; Bengio, Y. Generative Adversarial Nets. In Advances in Neural Information Processing Systems 27; Ghahramani, Z.; Welling, M.; Cortes, C.; Lawrence, N.D.; Weinberger, K.Q., Eds.; Curran Associates, Inc., 2014; pp. 2672-2680.

16. Mirza, M.; Osindero, S. Conditional Generative Adversarial Nets. CoRR 2014, abs/1411.1784.

17. Gauthier, J. Conditional generative adversarial networks for convolutional face generation. Technical report, Stanford University, 2015.

18. Cohen, G.; Afshar, S.; Tapson, J.; van Schaik, A. EMNIST: an extension of MNIST to handwritten letters. CoRR 2017, abs/1702.05373, [1702.05373].

19. Witthoft, N.; Winawer, J.; Eagleman, D.M. Prevalence of Learned Grapheme-Color Pairings in a Large Online Sample of Synesthetes. PLoS ONE 2015, 10.

20. Agrawal, M.; Sawhney, K. Exploring Convolutional Neural Networks for Automatic Image Colorization. Technical report, Stanford University, 2017.

21. Shwartz-Ziv, R.; Tishby, N. Opening the Black Box of Deep Neural Networks via Information. CoRR 2017, abs/1703.00810, [1703.00810].

22. Abadi, M.; Barham, P.; Chen, J.; Chen, Z.; Davis, A.; Dean, J.; Devin, M.; Ghemawat, S.; Irving, G.; Isard, M.; Kudlur, M.; Levenberg, J.; Monga, R.; Moore, S.; Murray, D.G.; Steiner, B.; Tucker, P.A.; Vasudevan, V.; Warden, P.; Wicke, M.; Yu, Y.; Zhang, X. TensorFlow: A system for large-scale machine learning. CoRR 2016, abs/1605.08695, [1605.08695].

23. Dixon, M.J.; Smilek, D.; Cudahy, C.; Merikle, P.M. Five plus two equals yellow. Nature 2000, 406, 365.

24. Mills, C.; Innis, J.; Westendorf, T.; Owsianiecki, L.; McDonald, A. Effect of a Synesthete's Photisms on Name Recall. Cortex 2006, 42, 155-63.

25. Crick, F.; Koch, C. A Framework for Consciousness. Nature Neuroscience 2003, 6, 119-26.

26. Hinton, G. Learning multiple layers of representation. Trends in Cognitive Sciences 2007, 11, $428-434$. 